\title{
Relationship Between Angiopoietin-Like Protein 8 and Fasting Serum Triglyceride Level
}

\author{
Hodaka Yamada ${ }^{\text {a, c }}$, Ikuyo Kusaka ${ }^{a}$, Rika Saikawa ${ }^{\mathrm{a}}$, Kazuo Hara ${ }^{\mathrm{a}}$, \\ Masafumi Kakei ${ }^{\mathrm{a}}$, San-e Ishikawa ${ }^{\mathrm{b}}$
}

\begin{abstract}
Background: The aim of the study was to evaluate the correlation between angiopoietin-like protein 8 (ANGPTL8) and metabolic parameters in non-diabetic healthy humans.

Methods: We enrolled 30 healthy Japanese adults (25 men and five women). After $9 \mathrm{~h}$ of fasting, we collected blood samples and analyzed the ANGPTL8, lipoprotein lipase (LPL), plasma lipid and glucose metabolic parameters. In addition, we performed 75-g oral glucose tolerance test (OGTT) and measured adipokines (tumor necrosis factor- $\alpha$, leptin and adiponectin).
\end{abstract}

Results: Median serum ANGPTL8 level was 224 (167 - 437) pg/mL, and serum ANGPTL8 level positively correlated with serum triglyceride level $(\mathrm{r}=0.42, \mathrm{P}=0.021)$ and negatively correlated with LPL level $(r=-0.44, P=0.015)$. ANGPTL8 level showed no correlation with body mass index (BMI), waist-hip ratio, and homeostasis model assessment of insulin resistance (HOMA-IR) or with adipose tissuederived adiponectin and leptin levels. Further, ANGPTL8 showed no association with glucose and insulin levels after 75-g OGTT.

Conclusion: Serum ANGPTL8 level negatively correlated with LPL levels in healthy Japanese adults. Regulation of ANGPTL8 could be a promising therapeutic target for hypertriglyceridemia.

Keywords: Angiopoietin-like protein 8; Hypertriglyceridemia; Lipoprotein lipase; Insulin resistance

\section{Introduction}

Angiopoietin-like protein 8 (ANGPTL8) is a lipid metabolic

Manuscript submitted November 30, 2017, accepted December 18, 2017

aFirst Department of Comprehensive Medicine, Division of Endocrinology and Metabolism, Jichi Medical University Saitama Medical Center, 1-847 Amanuma-cho, Omiya-ku, Saitama 330-8503, Japan

bivision of Endocrinology and Metabolism, International University of Health and Welfare Hospital, 537-3 Iguchi, Nasushiobara, Tochigi 329-2763, Japan

${ }^{\mathrm{c} C o r r e s p o n d i n g ~ A u t h o r: ~ H o d a k a ~ Y a m a d a, ~ D e p a r t m e n t ~ o f ~ M e d i c i n e, ~ J i c h i ~}$ Medical University Saitama Medical Center, 1-847 Amanuma-cho, Omiyaku, Saitama 330-8503, Japan. Email: hyamada0510@jichi.ac.jp

doi: https://doi.org/10.14740/jocmr3286w regulator derived from adipose tissues and liver [1]. ANGPTL8 (as betatrophin) is believed to control beta cell proliferation [2]. However, this finding could not be confirmed in subsequent replication studies [3, 4]. Phenotypical Angptl8 $8^{-/-}$mice exhibit low serum triglyceride (TG) levels [5], and it is thought that ANGPTL8/betatrophin is a crucial factor for TG metabolism [6]. Zhang et al originally revealed that male mice that overexpressed ANGPTL8 (with the use of an adenovirus vector) exhibited hypertriglyceridemia compared with controls overexpressing green fluorescent protein. In addition, they identified that recombinant ANGPTL8 inhibited lipoprotein lipase (LPL) activity [7]. In both mice and humans, some studies (including our previous report) showed that serum ANGPTL8 is elevated in individuals with diabetes and was positively correlated with TG levels $[8,9]$. However, the correlation between ANGPTL8 and metabolic parameters (i.e., adipokines and LPL) in nondiabetic healthy humans is unknown.

\section{Methods}

We enrolled 30 healthy Japanese adult volunteers ( 25 men and five women) who were not under any medications and had no significant past medical history. After $9 \mathrm{~h}$ of fasting, blood samples were collected into tubes from all subjects. The blood was centrifuged at $3,000 \mathrm{rpm}$ and $4{ }^{\circ} \mathrm{C}$ for 15 min to obtain serum, which was frozen at $-80^{\circ} \mathrm{C}$ until the time of assay.

We analyzed the following metabolic parameters and serum adipokine concentrations using commercially available ELISA kits: ANGPTL8 ELISA kit (Catalog No. E11644h; Wuhan Eiaab Science, Wuhan, China), LPL (Sekisui Medical Co. Ltd, Tokyo, Japan), C-reactive protein (CRP; EIA kit American Research Products, Belmont, MA, USA), tumor necrosis factor- $\alpha$ (TNF- $\alpha$; Invitrogen Corporation, CA, USA), leptin (R\&D Systems, Minneapolis, USA), and adiponectin (Fujirebio Inc., Tokyo, Japan). Other parameters were measured in the central laboratory section of the Jichi Medical University Saitama Medical Center using standard techniques. We performed a 75-g oral glucose tolerance test (OGTT) during morning fasting state and measured fasting plasma glucose (FPG) and fasting immunoreactive insulin (FIRI); 60 and 120 min later, we measured plasma glucose (PG) and immunoreactive insulin (IRI) levels, respectively. The homeostasis model assessment of insulin resistance (HOMA-IR, an index of insulin resistance) and HOMA- $\beta$ (an index of the capacity of insulin secretion in a fasting state) were calculated using the following equations: HOMA-IR $=(\mathrm{FPG} \times \mathrm{FIRI}) / 405$ and 
Table 1. Basic Clinical Parameters and Correlation Analysis With ANGPTL8

\begin{tabular}{llll}
\hline Parameters & Mean \pm SD or median & r & P-value \\
\hline Age $($ years $)$ & $51(44-56)$ & 0.295 & 0.114 \\
BMI $\left(\mathrm{kg} / \mathrm{m}^{2}\right)$ & $23.2 \pm 2.7$ & 0.291 & 0.118 \\
Waist-hip ratio & $0.87 \pm 0.05$ & 0.185 & 0.329 \\
FPG $(\mathrm{mg} / \mathrm{dL})$ & $97 \pm 6$ & -0.23 & 0.221 \\
FIRI $(\mu \mathrm{U} / \mathrm{mL})$ & $8.9(3.9-13.9)$ & 0.063 & 0.739 \\
PG60 $(\mathrm{mg} / \mathrm{dL})$ & $121 \pm 29$ & -0.284 & 0.128 \\
IRI60 $(\mu \mathrm{U} / \mathrm{mL})$ & $51(29-67)$ & -0.294 & 0.184 \\
PG120 $(\mathrm{mg} / \mathrm{dL})$ & $108 \pm 21$ & 0.092 & 0.630 \\
IRI120 $(\mu \mathrm{U} / \mathrm{mL})$ & $39(24-53)$ & 0.095 & 0.617 \\
HbA1c $(\%)$ & $5.1 \pm 0.2$ & 0.009 & 0.963 \\
TC $(\mathrm{mg} / \mathrm{dL})$ & $194(173-208)$ & 0.144 & 0.448 \\
TG $(\mathrm{mg} / \mathrm{dL})$ & $94(71-167)$ & 0.42 & 0.021 \\
HDL-C $(\mathrm{mg} / \mathrm{dL})$ & $52 \pm 10$ & -0.279 & 0.136 \\
LPL $(\mathrm{ng} / \mathrm{mL})$ & $1.65 \pm 0.45$ & -0.438 & 0.015 \\
HOMA- $\beta$ & $103(47-133)$ & 0.079 & 0.677 \\
HOMA-IR & $2.12(0.92-3.46)$ & 0.052 & 0.784 \\
CRP $(\mathrm{mg} / \mathrm{dL})$ & $0.04(0.03-0.07)$ & 0.154 & 0.416 \\
TNF- $\alpha(\mathrm{pg} / \mathrm{mL})$ & $0.53(0.48-0.72)$ & -0.186 & 0.325 \\
Leptin $(\mathrm{pg} / \mathrm{mL})$ & $26(12-35)$ & -0.023 & 0.903 \\
Adiponectin $(\mathrm{ng} / \mathrm{mL})$ & $9.5(5.6-16.2)$ & -0.127 & 0.503 \\
\hline H & & & \\
\hline
\end{tabular}

BMI: body mass index; FPG: fasting plasma glucose; FIRI: fasting immunoreactive insulin; HbA1c: glycated hemoglobin; TC: total cholesterol; TG: triglyceride; HDL-C: high-density lipoprotein cholesterol; LPL: lipoprotein lipase; HOMA- $\beta$ : homeostasis model assessment of $\beta$; HOMA-IR: homeostasis model assessment of insulin resistance;

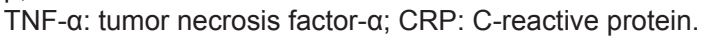

HOMA- $\beta=(360 \times \mathrm{FIRI}) /(\mathrm{FPG}-63)$.

Data are expressed as the mean \pm standard deviation (SD) or median with interquartile range. A Spearman correlation coefficient analysis was used to calculate correlations between serum ANGPTL8 and all dependent variables. All analyses were performed using EZR (Saitama Medical Center, Jichi Medical University), a graphical user interface for R (The R Foundation for Statistical Computing, ver. 2.13.0) and a modified version of the R Commander (ver. 1.6-3), which was designed to add frequently used biostatistical functions [10]. A threshold of $\mathrm{P}<0.05$ was considered significant. The study was approved by the Ethics Committee at Jichi Medical University Saitama Medical Center and performed in compliance with the Declaration of Helsinki. Written informed consent was obtained from all participants.

\section{Results}

Median participant age was 51 years and body mass index (BMI) was $23.2 \pm 2.7 \mathrm{~kg} / \mathrm{m}^{2}$. No subject exhibited abnormal glucose tolerance at baseline or following 75-g OGTT. Other clinical characteristics of the 30 adult participants are presented in Table 1. Median serum ANGPTL8 level was 224 (167 - 437) $\mathrm{pg} / \mathrm{mL}$, and there was no difference in serum ANGPTL8 between men and women (men 237 (164 - 442), women 210 (168 - 447), $\mathrm{P}=0.867)$, though only five women were included in a total of 30 participants. Serum ANGPTL8 level positively correlated with serum TG level $(\mathrm{r}=0.42, \mathrm{P}=0.021)$ and negatively correlated with LPL level $(r=-0.44, \mathrm{P}=0.015)$. ANGPTL8 level showed no correlation with BMI, waist-hip ratio, and HOMA-IR or with adipose tissue-derived adiponectin and leptin levels. In addition, ANGPTL8 showed no association with 75-g OGTT or with FPG and FIRI levels.

\section{Discussion}

We performed this study to clarify the relationship between circulating ANGPTL8 and lipid metabolic parameters, including LPL and adipokines. Our results demonstrated that fasting serum ANGPTL8 levels were closely associated with LPL in healthy adults. In an Angptl8 ${ }^{-/-}$mouse model, LPL activity increased and the uptake of very low-density lipoprotein-TG decreased in adipose tissue [5]. We do not assert a causal relationship between ANGPTL8 and LPL activity in this crosssectional study. A prospective cohort study revealed that fasting ANGPTL8 level positively correlated with fasting TG levels in 240 Korean children [11]. Our results were consistent with previous animal and human studies $[5,11]$. In contrast, a study reported that serum ANGPTL8 levels decreased in subjects with dyslipidemia [12] and negatively correlated with serum TG levels [13]. There is no clear evidence to explain these discrepancies. Fu et al reported that variability in ANGPTL8 measurement kits could affect results, namely the difference in $\mathrm{N}$-terminus-recognizing antibodies (full-length ANGPTL8) or C-terminus-recognizing antibodies (total ANGPTL8) [14]. We used the Eiaab N-terminus-recognizing ELISA kit in the present study. Previous studies have shown a positive relationship between circulating ANGPTL8 and TG levels using ELISA kits $[8,9,11]$. An ANGPTL8 assay system is needed to clarify whether full-length ANGPTL8 or fragmented ANGPTL8 truly reflects its bioactivity [8]. In humans, one study exhibited variable associations between ANGPTL8 (single nucleotide polymorphism, variant) and the serum lipid profile [6]. Peloso et al revealed that loss-of-function mutations of ANGPTL8 had low TG levels [15]. In the future, ANGPTL8 regulation might be a novel therapeutic option for targeting hypertriglyceridemia [8].

There were limitations to the present study. We used a cross-sectional design and examined a small cohort of participants. In addition, this study included deflection of the sex of the participant. Consequently, we cannot determine if elevated ANGPTL8 level is a causative factor. Large sample size study is also necessary. We did not analyze postprandial ANGPTL8 and lipid profiles. A previous study demonstrated elevated postprandial (2 $\mathrm{h}$ after a meal) ANGPTL8 levels [16]. In a fasting state, ANGPTL8 inhibited LPL of white adipose tissue and muscles, resulting in hypertriglyceridemia [6]. Further study is required to determine the postprandial lipid profile, including 
LPL and ANGPTL8.

Serum ANGPTL8 level negatively correlated with LPL levels in healthy Japanese adults. Regulation of ANGPTL8 could be a promising therapeutic target for hypertriglyceridemia.

\section{Conflict of Interest}

The authors declare that there is no conflict of interest.

\section{Funding}

None.

\section{References}

1. Dijk W, Kersten S. Regulation of lipid metabolism by angiopoietin-like proteins. Curr Opin Lipidol. 2016;27(3):249-256.

2. Yi P, Park JS, Melton DA. Betatrophin: a hormone that controls pancreatic beta cell proliferation. Cell. 2013;153(4):747-758.

3. Gusarova V, Alexa CA, Na E, Stevis PE, Xin Y, Bonner-Weir S, Cohen JC, et al. ANGPTL8/betatrophin does not control pancreatic beta cell expansion. Cell. 2014;159(3):691-696.

4. Jiao Y, Le Lay J, Yu M, Naji A, Kaestner KH. Elevated mouse hepatic betatrophin expression does not increase human beta-cell replication in the transplant setting. Diabetes. 2014;63(4):1283-1288.

5. Wang Y, Quagliarini F, Gusarova V, Gromada J, Valenzuela DM, Cohen JC, Hobbs HH. Mice lacking ANGPTL8 (Betatrophin) manifest disrupted triglyceride metabolism without impaired glucose homeostasis. Proc Natl Acad Sci U S A. 2013;110(40):16109-16114.

6. Zhang R. The ANGPTL3-4-8 model, a molecular mechanism for triglyceride trafficking. Open Biol. 2016;6(4):150272.

7. Zhang R. Lipasin, a novel nutritionally-regulated liver- enriched factor that regulates serum triglyceride levels. Biochem Biophys Res Commun. 2012;424(4):786-792.

8. Yamada H, Saito T, Aoki A, Asano T, Yoshida M, Ikoma A, Kusaka I, et al. Circulating betatrophin is elevated in patients with type 1 and type 2 diabetes. Endocr J. 2015;62(5):417-421.

9. Gao T, Jin K, Chen P, Jin H, Yang L, Xie X, Yang M, et al. Circulating betatrophin correlates with triglycerides and postprandial glucose among different glucose tolerance statuses - a case-control study. PLoS One. 2015;10(8):e0133640.

10. Kanda Y. Investigation of the freely available easy-touse software 'EZR' for medical statistics. Bone Marrow Transplant. 2013;48(3):452-458.

11. Chung HS, Lee MJ, Hwang SY, Lee HJ, Yoo HJ, Seo JA, Kim SG, et al. Circulating angiopoietin-like protein 8 (ANGPTL8) and ANGPTL3 concentrations in relation to anthropometric and metabolic profiles in Korean children: a prospective cohort study. Cardiovasc Diabetol. 2016;15:1.

12. Gomez-Ambrosi J, Pascual-Corrales E, Catalan V, Rodriguez A, Ramirez B, Romero S, Vila N, et al. Altered concentrations in dyslipidemia evidence a role for ANGPTL8/Betatrophin in lipid metabolism in humans. J Clin Endocrinol Metab. 2016;101(10):3803-3811.

13. Gomez-Ambrosi J, Pascual E, Catalan V, Rodriguez A, Ramirez B, Silva C, Gil MJ, et al. Circulating betatrophin concentrations are decreased in human obesity and type 2 diabetes. J Clin Endocrinol Metab. 2014;99(10):E20042009.

14. Fu Z, Abou-Samra AB, Zhang R. An explanation for recent discrepancies in levels of human circulating betatrophin. Diabetologia. 2014;57(10):2232-2234.

15. Peloso GM, Auer PL, Bis JC, Voorman A, Morrison AC, Stitziel NO, Brody JA, et al. Association of low-frequency and rare coding-sequence variants with blood lipids and coronary heart disease in 56,000 whites and blacks. Am J Hum Genet. 2014;94(2):223-232.

16. Fu Z, Berhane F, Fite A, Seyoum B, Abou-Samra AB, Zhang R. Elevated circulating lipasin/betatrophin in human type 2 diabetes and obesity. Sci Rep. 2014;4:5013. 